\title{
ROBUST STABILIZATION OF DISCRETE-TIME MULTI-MODEL SYSTEMS USING PIECEWISE AFFINE STATE-FEEDBACK
}

\author{
Olav Slupphaug * Bjarne A. Foss ${ }^{*, 1}$ \\ * Norwegian University of Science and Technology \\ Department of Engineering Cybernetics \\ email: Bjarne.Foss@itk.ntnu.no
}

\begin{abstract}
In this paper we develop a BMI based method for nonlinear robust stabilization. Robustness against model uncertainty is handled. The development is based on an uncertain multi-model representation of the plant, and an associated piecewise affine state-feedback structure. Assuming a quadratic Liapunov function, a BMI condition for robust (quadratic) stabilization is found. Control constraints are formulated as BMIs or LMIs. A branch-and-bound algorithm is used for solving the BMI problem, that is, finding the unknown quadratic Liapunov function and the piecewise affine state-feedback. Finally, an example is given.
\end{abstract}

Keywords: Nonlinear systems, discrete-time, quadratic stabilization, multi-model systems, BMI, constraints

\section{INTRODUCTION}

Robust controller design is a key factor for implementing controllers. Robust design becomes particularly important, but also challenging, for nonlinear uncertain systems, the outset for this work.

In our approach we have utilized results and ideas from LMI based control (Boyd et al., 1994), (Johansson and Rantzer, 1997), and (Petterson and Lennartson, 1997), and multimodel systems (Murray-Smith and Johansen, 1997).

In recent years much work has been put into the development of nonlinear models which are composed of a set of local models (MurraySmith and Johansen, 1997). The local models are valid in different parts of some predefined operating set in which the operating point typically will be defined by some of the measured control inputs and/or system outputs. Further, the nonlinear (global) model is formed as a convex combination of the local models. Other names for multi-model systems are operating regime based models and fuzzy models. In this work we focus on multi-model systems in which the local models are affine discrete-time statespace models, and we utilize this model structure to describe the model uncertainty class. This structure has at least three important advantages: (i) It is possible to utilize the affine structure of the local models for analysis and synthesis; (ii) the model class is rich in the sense that it approximates arbitrarily close a very large class of nonlinear systems; (iii) the model structure is transparent and there exist support tools for model identification (Johansen and Foss, 1997).

In LMI-based control, which also has gained a lot of interest in the last few years, control system analysis- and synthesis problems are formulated as convex optimization problems involving linear matrix inequalities (LMIs). The reason for this interest is the development of very efficient interior point algorithms for solving such problems (Boyd et al., 1994). Many interesting control problems, in particular robust control problems, can be solved within the LMI framework. There are, however, interesting control problems which are very hard or impossible to formulate within the LMI framework. Some of these problems can be formulated within the more general bilinear matrix inequalities (BMIs) framework (Goh et al., 1994). We utilize a piecewise affine state-feedback structure coupled with the uncertain multi-model to formulate the robust constrained nonlinear stabilization problem as a BMI feasibility problem. BMI problems are much harder than LMI problems since they, in general, are nonconvex. The efficient algorithms developed for the LMI problems do, however, provide a constructive basis on which branch-and-bound algorithms for BMI problems can be developed, see (Goh et al., 1994), (Tuan et al., 1997), and (Kawanishi et al., 1997).

1 Corresponding author 
with other approaches as shown in (Slupphaug and Foss, 1998a). In that paper the method developed herein constitutes a basis for developing a robust MPC scheme for constrained uncertain nonlinear systems.

The paper is organized as follows. Firstly, we present the considered multi-model uncertainty class. Then a BMI is found, which, if it is feasible, guarantees robust constrained stabilization of the origin of the uncertain system. Before the conclusion, the solution of the BMI feasibility problem is discussed, and an example is provided.

Some notation: $I_{M}:=\{1, \ldots, M\} ; N(x)$ is a neighbourhood of $x ; P>0(P \geq 0)$ implies $P=P^{T} ;\|x\|_{H}^{2}:=x^{T} H x$ where $H>0$; let $a, b \in \mathbb{N}$ then $\{a, \ldots, b\}:=\emptyset$ and $\left\{c_{l}\right\}_{l=a}^{b}:=\emptyset$, when $b<a$.

\section{PROBLEM STATEMENT AND UNCERTAINTY MODEL}

The problem we investigate is to find a statefeedback which robustly stabilize the origin of a plant which can be described by a convex combination of affine discrete-time state-space systems. That is, the uncertain (nonlinear) plant is assumed to be given by

$$
x_{k+1}=\sum_{j \in I_{N_{m}}} \omega_{j}\left(x_{k}, u_{k}, k\right)\left(A_{j} x_{k}+B_{j} u_{k}+c_{j}\right),
$$

where $k \geq 0, x_{0}$ given, $x_{k} \in X_{m} \subset \mathbb{R}^{n}$, $u_{k} \in U_{m} \subset \mathbb{R}^{m}$, the local models $\left(A_{j}, B_{j}, c_{j}\right)$ 's are triplets which elements have appropriate dimensions, $N_{m}$ is the number of local models (subscript $m$ indicates "model"),

$$
\omega_{j}: X_{m} \times U_{m} \times \mathbb{N} \rightarrow[0,1], \forall j \in I_{N_{m}},
$$

and

$$
\sum_{j \in I_{N_{m}}} \omega_{j}(x, u, k)=1, \forall(x, u, k) \in X_{m} \times U_{m} \times \mathbb{N} .
$$

The model validity sets $X_{m}$ and $U_{m}$ are assumed to be connected sets containing the origin in their interior.

Uncertainty is represented by allowing $\omega(\cdot, \cdot, \cdot):=$ $\left(\omega_{1}(\cdot, \cdot, \cdot), \ldots, \omega_{N_{m}}(\cdot, \cdot, \cdot)\right)$ to vary within a predefined set $\Omega$. Next, this set will be defined.

The uncertainty description is based on the assumption that all that is known about the weights are their state-space supports, $X_{j}^{S}$ (superscript $S$ indicates "support"), i.e., knowledge of the sets

$$
X_{j}^{S}:=\bigcup_{(u, k) \in U_{m} \times \mathbb{N}}\left\{x \mid \omega_{j}(x, u, k)>0\right\}, \forall j \in I_{N_{m}} .
$$

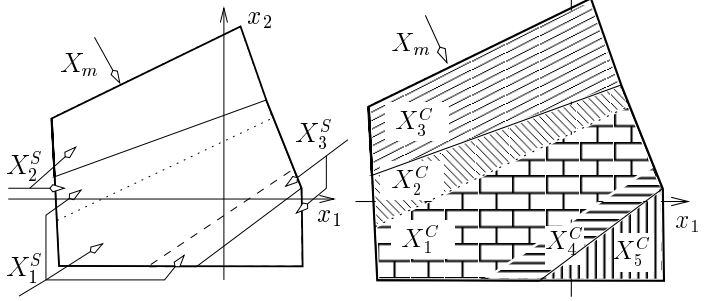

Fig. 1. The state-space supports; $X_{1}^{S}, X_{2}^{S}$, and $X_{3}^{S}$ for a multi-model system with three local models (left), and the associated 5 clusters (right).

We notice that the projection on the state-space for all $u \in U_{m}$ in Eq. (3) implies that nonlinearities associated with the control input will be conservatively handled. It should be noted, however, that an arbitrary nonlinearity associated with the control input can be handled.

Associated with the state-space supports we define the following sets: for all $j \in I_{N_{m}}$

$$
\begin{aligned}
\Omega_{j} & :=\left\{\tilde{\omega} \mid \tilde{\omega}: X_{m} \times U_{m} \times \mathbb{N} \rightarrow[0,1]\right. \\
& \text { and } \left.\tilde{\omega}(x, u, k)>0 \text { only when } x \in X_{j}^{S}\right\},
\end{aligned}
$$

i.e. the set of all possible weights for local model number $j$. Now, let

$$
\begin{gathered}
\Omega:=\left\{\omega=\left(\omega_{1}, \ldots, \omega_{N_{m}}\right) \in \Omega_{1} \times \ldots \times \Omega_{N_{m}} \mid\right. \\
\left.\sum_{j \in I_{N_{m}}} \omega_{j}(x, u, k)=1, \forall(x, u, k) \in X_{m} \times U_{m} \times \mathbb{N}\right\},
\end{gathered}
$$

i.e. the set of all valid convex combinations, and $f_{\omega}(x, u, k):=\sum_{j \in I_{N_{m}}} \omega_{j}(x, u, k)\left(A_{j} x+B_{j} u+c_{j}\right)$.

Finally

$$
\mathcal{M}:=\left\{f_{\omega} \mid \omega \in \Omega\right\} .
$$

Thus, $\mathcal{M}$ now denotes the assumed multi-model uncertainty class.

Local models with $c_{j} \neq 0$ are assumed not to have support in some neighborhood of the origin. This amounts to assuming that all the plants $f \in \mathcal{M}$, and in particular the real plant, satisfies $0=f(0,0, k)$ for all $k \geq 0$, i.e. the equilibrium state and -control input are assumed to be known.

With the state-space supports, $X_{j}^{S}$, we also associate a partitioning of the state space into a set of $N_{c}$ clusters. A cluster, $X_{j}^{C}$, is a set on which the same local models have support on the whole set, and if it is extended, at least one of the local models will not have support on the extension. With each cluster, $X_{j}^{C}$, we associate a set of local models, or, more precisely, a set of local model numbers $\Lambda_{j}$. In Figure 1 (right part) the 5 clusters associated with the state-space supports given in Figure 1 (left part) are shown, in this case; $\Lambda_{1}=\{1\}, \Lambda_{2}=\{1,2\}, \Lambda_{3}=\{2\}$, $\Lambda_{4}=\{1,3\}$ and, $\Lambda_{5}=\{3\}$.

We will let $X_{m}$ and $U_{m}$ denote the state- and control constraints, respectively. In what follows 
however, have been any connected subsets of, respectively, $X_{m}$ and $U_{m}$ containing the origin in their interior.

\section{ROBUST STABILIZATION}

The aim of this section is to provide computationally verifiable sufficient conditions for robust stabilizability of the origin of system (1). The outcome of the computation will, when it is successful, be a piecewise affine state-feedback controller, and a quadratic Liapunov function for the origin of the closed-loop.

\subsection{Piecewise Affine State-Feedback}

We finitely parameterize the state-feedback, $u(x)$, as a piecewise affine state-feedback. With the cluster containing the origin and the clusters which closure contains the origin, assumed (without loss of generality) to be the first $N_{c}^{o}$ clusters, we associate a linear state feedback

$$
u(x)=K_{l} x \text { when } x \in X_{l}^{C}, l \in I_{N_{c}^{o}} .
$$

With all the other clusters we associate an affine state feedback, i.e. for $l \in\left\{N_{c}^{o}+1, \ldots, N_{c}\right\}$

$$
u(x)=K_{l} x+k_{l} \text { when } x \in X_{l}^{C} .
$$

Remember that the clusters form a partition of $X_{m}$, so the above defined piecewise affine statefeedback is indeed well defined.

It should be noted that there is, in principle, no problem associating the piecewise statefeedback with a different partitioning of $X_{m}$ than the one associated with the clusters. For reasons of clarity, however, we restrict the piecewise affine state-feedback to be associated with the clusters.

\subsection{Set Approximations}

To get BMI conditions for robust (constrained) stabilization we need to approximate the clusters, and state- and control constraints using polytopes or ellipsoids. A short discussion on the outer approximations below is given in (Slupphaug and Foss, 1998b). Here, we only note that the given approximations exist for the given sets, and save them for later reference.

Assume that for $l \in I_{N_{c}}$ the polytope

$$
\left\{x \mid E_{l} x \leq 0\right\} \supset X_{l}^{C}
$$

is used as an outer approximation of $X_{l}^{C}$. For $l \in\left\{N_{c}^{o}+1, \ldots, N_{c}^{p}\right\}$ assume that the polytope

$$
\left\{x \mid\left[E_{l} e_{l}\right]\left[\begin{array}{l}
x \\
1
\end{array}\right] \leq 0\right\} \supset X_{l}^{C}
$$

assume that the ellipsoid

$$
\left\{x \mid\left[\begin{array}{c}
x \\
1
\end{array}\right]^{T}\left[\begin{array}{cc}
E_{l} & e_{l} \\
e_{l}^{T} & \epsilon_{l}
\end{array}\right]\left[\begin{array}{l}
x \\
1
\end{array}\right] \leq 0\right\} \supset X_{l}^{C}
$$

is used. $N_{c}^{p}$ is the number of clusters outer approximated by polytopes. Furthermore, assume that $X_{m}$ is inner approximated as follows

$$
\bigcap_{i \in I_{N_{q x}}}\left\{x \mid\left\|x-x_{i, c}\right\|_{H_{i, x}}^{2} \leq 1\right\} \subset X_{m},
$$

i.e. by an intersection of ellipsoids where $x_{i, c}$ denotes the centers of the ellipsoids, and $N_{q x}$ denotes the number of ellipsoids. Similarly, we assume

$$
\bigcap_{i \in I_{N_{q u}}}\left\{u \mid\left\|u-u_{i, c}\right\|_{H_{i, u}}^{2} \leq 1\right\} \subset U_{m} .
$$

\subsection{BMI for Robust Stabilization}

In this subsection, we investigate quadratic stability of the origin of the closed-loop using the piecewise affine state-feedback defined by Eqs. (4). We will let $U_{m}=\mathbb{R}^{m}$, i.e. it assumed that no input constraints are present. The constrained case is deferred to Section 5.

First, we precisely define quadratic stability in the present context. Based on (Corless, 1994) the following definition is adopted.

\section{DEFINITION 1}

Given an uncertain system

$$
\begin{aligned}
x_{k+1} & =f\left(x_{k}, k\right) \\
f & \in \tilde{\mathcal{M}}
\end{aligned}
$$

where $k \geq 0, x_{k} \in \mathbb{R}^{n}, x_{0}$ given, and all $f \in \tilde{\mathcal{M}}$ satisfies: $f: \tilde{X}_{m} \times \mathbb{N} \rightarrow \mathbb{R}^{n}$ and $f(0, k)=0$ for all $k \geq 0$. We say that the origin is a quadratically stable equilibrium for system (10) if there $\exists M, P>0, N(0)$ such that $N(0) \subset \tilde{X}_{m}$ and $\forall(a, i) \in N(0) \times \mathbb{N}$

$$
f(a, i)^{T} P f(a, i)-a^{T} P a \leq-a^{T} M a,
$$

If, in addition, there exists $\alpha \in(0, \infty)$ such that for a given set $\tilde{R}_{A}$

$$
\tilde{R}_{A} \subset\left\{x \mid x^{T} P x \leq \alpha\right\} \subset N(0)
$$

then the origin is said to be a quadratically stable equilibrium for system (10) with a region of attraction associated with $\tilde{R}_{A}$ of at least $\left\{x \mid x^{T} P x \leq \alpha\right\}$.

Note that quadratic stability implies robust exponential stability.

Next, the main result is presented.

THEOREM 1

If, restricting the $W_{l}$ 's to be symmetric and have nonnegative elements, there $\exists M>0, P=$ $P^{T}, S=S^{T},\left\{K_{l}\right\}_{l=1}^{N_{c}},\left\{k_{l}\right\}_{l=N_{c}^{o}+1}^{N_{c}},\left\{W_{l}\right\}_{l=1}^{N_{c}^{p}}$, 
$\forall l \in I_{N_{c}^{o}}, j \in \Lambda_{l}$,

$$
\left[\begin{array}{cc}
S & A_{j}+B_{j} K_{l} \\
\star & P-M-E_{l}^{T} W_{l} E_{l}
\end{array}\right] \geq 0
$$

$\forall l \in\left\{N_{c}^{o}+1, \ldots, N_{c}^{p}\right\}, j \in \Lambda_{l}$

$$
\left[\begin{array}{ccc}
S & A_{j}+B_{j} K_{l} & B_{j} k_{l}+c_{j} \\
\star & P-M-E_{l}^{T} W_{l} E_{l} & -E_{l}^{T} W_{l} e_{l} \\
\star & \star & -e_{l}^{T} W_{l} e_{l}
\end{array}\right] \geq 0
$$

$\forall l \in\left\{N_{c}^{p}+1, \ldots, N_{c}\right\}, j \in \Lambda_{l}$,

$$
\left[\begin{array}{ccc}
S & A_{j}+B_{j} K_{l} & B_{j} k_{l}+c_{j} \\
\star & P-M+\tau_{l} E_{l} & \tau_{l} e_{l} \\
\star & \star & \tau_{l} \epsilon_{l}
\end{array}\right] \geq 0
$$

and

$$
S P+P S \leq 2 I,
$$

then the origin is a quadratically stable equilibrium for the closed-loop.

If, in addition, there exist reals $\alpha,\left\{\lambda_{i}\right\}_{i \in I_{N_{q x}}}$, and $\beta$ such that

$$
\begin{aligned}
& {\left[\begin{array}{cc}
P-\beta R_{A} & 0 \\
0 & \beta-\alpha
\end{array}\right] } \leq 0, \\
&(13 \mathrm{a}) \\
& {\left[\begin{array}{cc}
\lambda_{i} H_{i, x}-P & -\lambda_{i} H_{i, x} x_{i, c}, \\
\star & \lambda_{i}\left(x_{i, c}^{T} H_{i, x} x_{i, c}-1\right)+\alpha
\end{array}\right] } \leq 0, \\
&(13 \mathrm{~b}
\end{aligned}
$$

then the origin is a quadratically stable equilibrium for the closed-loop with a region of attraction associated with $\left\{x\|\| x \|_{R_{A}}^{2} \leq 1\right\}$ of at least $\left\{x \mid x^{T} P x \leq \alpha\right\}$.

$\triangle$

The $\star$ elements are induced by symmetry of the associated matrices. The given set $\left\{x\|\| x \|_{R_{A}}^{2} \leq\right.$ $1\}$ would typically denote the smallest acceptable region of attraction.

The proof (Slupphaug and Foss, 1998b) proceeds by using the so-called $\mathcal{S}$-procedure and Schur complements (Boyd et al., 1994), and some other results on matrix inequalities.

The LMIs (11) are conditions for decrease of the Liapunov function along closed-loop trajectories in the different clusters. The LMIs (13) are conditions for the Liapunov level set $\left\{x \mid x^{T} P x \leq \alpha\right\}$ to contain $\left\{x \mid\|x\|_{R_{A}}^{2} \leq 1\right\}$ (13a), while simultaneously being contained in $X_{m}$ (13b). The BMI (12) origins from the inequality $P^{-1} \geq S$, which emanates from using Schur complements to get the LMIs (11). The core of the problem with getting an equivalent condition involving only LMIs, which of course would be much more attractive from a computationally point of view, is the indefiniteness of the $\mathcal{S}$ procedure terms, which seems to necessarily call for a BMI formulation. It is this indefinitness, different local models and state-feedbacks with different parts of the state-space, thus removing it will definitely introduce significant conservatism. Lastly, it should be noted that a, in general very conservative, LMI transformation of the BMI in Theorem 1 exist. This can be done by forcing $S=P$ and replacing the BMI (12) by the LMI

$$
\left[\begin{array}{cc}
2(I+P) & P+I \\
\star & I
\end{array}\right] \geq 0,
$$

which stems from $P^{-1} \geq P$. To illustrate why this may be very conservative take $P \in$ $\mathbb{R}^{2 \times 2}$, then, for $P^{-1} \geq$ $P$ and $P>$ 0 to be satisfied, the elements of $P$ must lie between the two surfaces in Figure 2, whereas $P>0$ for any point

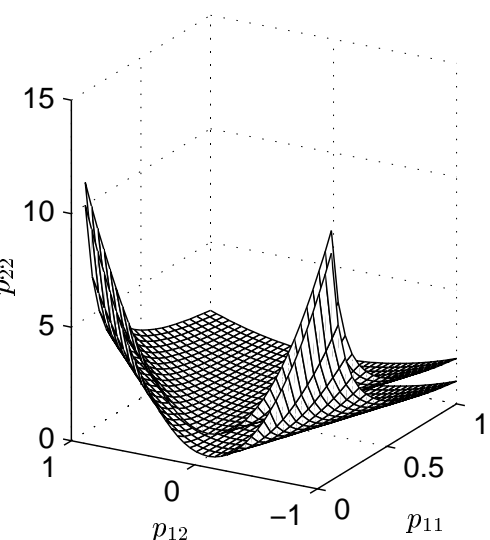
above the lowest surface.

\section{BRANCH-AND-BOUND FOR THE BMI FEASIBILITY PROBLEM}

With the BMI feasibility problem associated with Theorem 1 we associate the following eigenvalue optimization problem denoted $\mathcal{P}_{\mathrm{EV}}$ :

$$
\min \vartheta
$$

subject to

$$
\begin{aligned}
M & >-\vartheta I, \\
\mathcal{A}\left(P, S, M,\left\{K_{l}\right\}_{l=1}^{N_{c}},\left\{k_{l}\right\}_{l=N_{c}^{o}+1}^{N_{c}}, \ldots\right) & \geq-\vartheta I, \\
\mathcal{B}(P, S) & \geq-\vartheta I .
\end{aligned}
$$

The minimization is over all matrix and scalar variables in the matrix inequalities. The affine symmetric matrix valued mapping $\mathcal{A}(\cdot, \ldots, \cdot)$ is given by (11), (13) and the nonnegativity condition on the elements of the $W_{l}$ 's, and the biaffine symmetric matrix valued mapping $\mathcal{B}(\cdot, \cdot)$ is given by $(12)$.

It is clear that if $\vartheta^{*}<0$, where $\vartheta^{*}$ is the value of $\vartheta$ at the optimum, then, and only then, the BMI and LMIs in Theorem 1 is satisfiable. $\mathcal{P}_{\mathrm{EV}}$ is a biconvex non-smooth optimization problem (Goh et al., 1994).

We use branch-and-bound algorithm 3 in (Tuan et al., 1997) for solving $\mathcal{P}_{\mathrm{EV}}$ (of course we 
algorithm 3 the branching is done on a set of lower dimension, in our case much lower, than the total problem dimension, as opposed to (Goh et al., 1994) and (Kawanishi et al., 1997) where the branching is done on a set with dimension equal to the total problem size. The number of so-called complicating variables gives the dimension of this lower dimensional set. The number of complicating variables is the smallest number of variables that need to be fixed to make the BMI an LMI. In our case the BMI structure arises due to the single BMI (12) (when control constraints are represented as LMIs). Since $P=P^{T} \in \mathbb{R}^{n \times n}$, this gives $\left(n^{2}+n\right) / 2$ complicating variables (the number of independent elements in $P$ ) which is much lower than the total problem size which might be ten times the number of complicating variables.

The lower bounding in algorithm 3 is done by a tight relaxation of the BMI (12) to an LMI and then the resultant LMI problem is solved, while the upper bounding is done by solving an eigenvalue problem which is parameterized by the solution of the lower bounding problem. The branching is done so as to force the difference between the relaxed BMI and the original BMI "quickly" towards zero.

\section{CONTROL INPUT CONSTRAINTS AS BMIS OR LMIS}

To satisfy the control constraints, $U_{m}$, on all possible closed-loop trajectories starting within $\left\{x\|\| x \|_{R_{A}}^{2} \leq 1\right\}$ it is sufficient to satisfy them on the positively invariant embracing set $\left\{x \mid x^{T} P x \leq \alpha\right\} \subset X_{m}$. For this to be the case it is sufficient that for all $(i, l) \in I_{N_{q u}} \times I_{N_{c}}$ and for all $x \in X_{l}^{C} \cap\left\{x \mid x^{T} P x \leq \alpha\right\}$

$$
\left\|K_{l} x+k_{l}-u_{i, c}\right\|_{H_{i, u}}^{2} \leq 1 \text {. }
$$

When outer approximating the clusters $X_{l}^{C}$ in connection with formulating BMI or LMI conditions for satisfying control constraints one can use anyone of the approximations (5), (6), or (7). One is not restricted to use (5) when $l \in I_{N_{c}^{o}}$. We present the results when using $(7)$, similar results for the other two cases can be derived. In this case we get, by invoking the $\mathcal{S}$-procedure followed by using Schur complements, that (14) can be transformed into the following sufficient BMI condition

$$
\left[\begin{array}{ccc}
\tau_{l, i}^{P} P+\tau_{l, i}^{E} E_{l} & \tau_{l, i}^{E} e_{l} & K_{l}^{T} \\
\star & 1-\tau_{l, i}^{P} \alpha+\tau_{l, i}^{E} \epsilon_{l} & k_{l}-u_{i, c}^{T} \\
\star & \star & H_{i, u}^{-1}
\end{array}\right] \geq 0 .
$$

where all the (scalar) $\tau$ 's must be nonnegative, and $k_{l}:=0$ when $l \in I_{N_{c}^{o}}$. Notice that this results in only one more complicating variable tive) LMI condition for satisfaction of the control constraints (Slupphaug and Foss, 1998b).

\section{EXAMPLE}

In this example we show that the proposed design procedure provides stabilizing controllers for multi-model systems where there are uncontrollable local models, as well as local models which are affine and not merely linear. In particular, having uncontrollable local models means that no controller can simultaneously stabilize all the local models on the whole state-space.

The uncertain multi-model consists of three affine local models, and has three clusters. The local models are as follows

$$
\begin{aligned}
& A_{1}=\left[\begin{array}{cc}
-1 & 2 \\
-1.2 & 1
\end{array}\right] B_{1}=100\left[\begin{array}{ll}
.1 & 0 \\
.1 & 1
\end{array}\right] c_{1}=\left[\begin{array}{l}
0 \\
0
\end{array}\right], \\
& A_{2}=\left[\begin{array}{cc}
-1.1 & 0 \\
.3 & -1.1
\end{array}\right] B_{2}=100\left[\begin{array}{ll}
1 & 0 \\
0 & 0
\end{array}\right] c_{2}=\left[\begin{array}{l}
1 \\
0
\end{array}\right], \\
& A_{3}=\left[\begin{array}{cc}
1.5 & 0 \\
.4 & -.7
\end{array}\right] B_{3}=100\left[\begin{array}{ll}
0 & 0 \\
0 & 1
\end{array}\right] c_{3}=\left[\begin{array}{l}
0 \\
1
\end{array}\right] .
\end{aligned}
$$

Notice that all local models are open-loop unstable. Furthermore local model number 3 is not controllable. In this example we let $U_{m}=$ $[-1,1] \times[-1,1]$, and $X_{m}=\left\{x \mid x_{1}^{2}+x_{2}^{2}-25 \leq 0\right\}$. $X_{m}$, being itself an ellipsoid, is equal to its inner approximation. $U_{m}$ is inner approximated using two ellipsoids with centers at the origin and with longest axes ten times the length of the shortest.

The clusters and sets of associated local models are

$$
\begin{aligned}
X_{1}^{C} & =X_{m} \backslash\left(X_{2}^{C} \cup X_{3}^{C}\right), \\
X_{2}^{C} & =\left\{x \mid\left(x_{1}-3\right)^{2}+\left(x_{2}-3\right)^{2}-1 \leq 0\right\}, \\
X_{3}^{C} & =\left\{x \mid\left(x_{1}+3\right)^{2}+\left(x_{2}+3\right)^{2}-1 \leq 0\right\}, \\
\Lambda_{1} & =\{1\}, \Lambda_{2}=\{1,2\}, \Lambda_{3}=\{1,3\} .
\end{aligned}
$$

$X_{1}^{C}$ is outer approximated by $X_{m}$, while $X_{2}^{C}$ and $X_{3}^{C}$ equal their outer approximations.

Applying the developed BMI based robust design strategy to this example system, we get the following piecewise affine state feedback controller

$$
\begin{aligned}
K_{1} & =\left[\begin{array}{ll}
0.0637 & -0.0746 \\
0.0061 & -0.0043
\end{array}\right], \\
K_{2} & =\left[\begin{array}{ll}
0.0117 & 0.0010 \\
0.0121 & -0.0129
\end{array}\right] k_{2}=\left[\begin{array}{c}
-0.0094 \\
0.0011
\end{array}\right], \\
K_{3} & =\left[\begin{array}{cc}
0.1459 & -0.1554 \\
-0.0051 & 0.0060
\end{array}\right] k_{3}=\left[\begin{array}{c}
0.0666 \\
-0.0087
\end{array}\right],
\end{aligned}
$$

and Liapunov matrix

$$
P=\left[\begin{array}{ll}
0.8760 & 0.2634 \\
0.2634 & 1.8861
\end{array}\right]
$$

accompanied by $\alpha=73.8766$. 
closed-loop motions ('o' and ' $x$ ') is given in the left part of Figure 3 ('o' starts at $x \approx(-9,4.5)^{T}$ and ' $\mathrm{x}$ ' starts at $\left.x \approx(3.5,3.5)^{T}\right)$. We observe that the closed loop trajectories are confined to the set $\left\{x \mid x^{T} P x \leq \alpha\right\}$ when starting inside it, and that the prescribed relationship $\left\{x\||| x\|_{R_{A}} \leq 1\right\} \subset\left\{x \mid x^{T} P x \leq \alpha\right\} \subset X_{m}$ is satisfied (the borders of each of these sets are shown). In the right part of Figure 3 we observe that the Liapunov function is indeed decreasing, and that the control input constraints are satisfied (these plots show the 'o' simulation). In the simulations the following weights defined the real system

$$
\begin{aligned}
& \omega_{1}=1 \text { on } X_{1}^{C}, 0.2 \text { on } X_{2}^{C}, \text { and } 0.6 \text { on } X_{3}^{C}, \\
& \omega_{2}=0 \text { on } X_{1}^{C}, 0.8 \text { on } X_{2}^{C}, \text { and } 0 \text { on } X_{3}^{C}, \\
& \omega_{3}=0 \text { on } X_{1}^{C}, 0 \text { on } X_{2}^{C}, \text { and } 0.4 \text { on } X_{3}^{C} .
\end{aligned}
$$
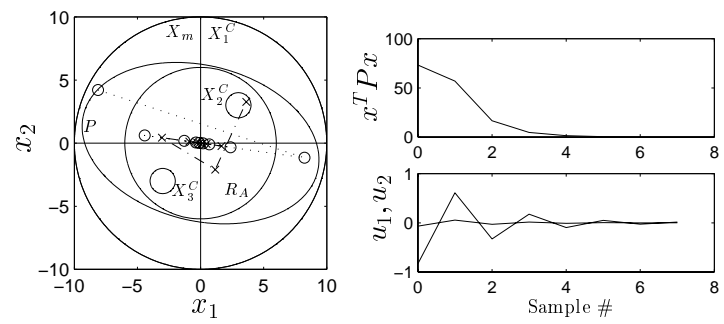

Fig. 3. Simulation results.

\section{DISCUSSION AND CONCLUSIONS}

We note that important problems such as output feedback and disturbance rejection cannot, at the moment, be addressed by the given design procedure. However, the work (Dussy and El Ghaoui, 1997) provides hope for such extensions.

In conclusion, a computationally solvable robust constrained controller synthesis method is developed. Robustness against model uncertainty is investigated, and the problem solved is a stabilization problem.

\section{ACKNOWLEDGEMENTS}

We acknowledge the useful comments from Mr. Georgo Angelis at Eindhoven University of Technology, The Netherlands, and the thorough review of reviewer \# 20.

\section{REFERENCES}

Boyd, Stephen, Laurent El Ghaoui, Eric Feron and Venkataramanan Balakrishnan (1994). Linear Matrix Inequalities in System and Control Theory. number 15 In: SIAM Studies in Applied Mathematics. SIAM. ysis and Controller Design with Quadratic Lyapunov Functions. In: Variable Structure and Lyapunov Control (Alan S. I. Zinober, Ed.). Chap. 9. Number 193 In: Lecture Notes in Control and Information Sciences. Springer-Verlag. London.

Dussy, Stéphane and Laurent El Ghaoui (1997). Multiobjective Bounded Control of Uncertain Nonlinear Systems: An Inverted Pendulum Example. In: Proceedings of ECC'97, Bruxelles, Belgium.

Goh, K. C., M. G. Safonov and G. P. Papavassilopoulos (1994). A Global Optimization Approach for the BMI Problem. In: Proceedings of the 33rd CDC, Lake Buena Vista, FL, USA. pp. 2009-14.

Johansen, T. A. and B. A. Foss (1997). ORBIT - operating regime based modeling and identification toolkit. In: Preprints IFAC Symposium on System Identification, Kitakyushu, Japan. pp. 961-968.

Johansson, Mikael and Anders Rantzer (1997). Computation of Piecewise Quadratic Lyapunov Functions for Hybrid Systems. In: Proceedings of ECC'97, Bruxelles, Belgium.

Kawanishi, Michihiro, Toshiharu Sugie and Hiroshi Kanki (1997). BMI Global Optimization Based on Branch and Bound Method Taking Account of The Property of Local Minima. In: Proceedings of the 36th Conference on Decision and Control. Vol. 1. San Diego, CA, USA. pp. 781-86.

Murray-Smith, R. and Johansen, T. A., Eds.) (1997). Multiple Model Approaches to Modelling and Control. Taylor and Francis. London.

Petterson, Stefan and Bengt Lennartson (1997). An LMI Approach for Stability Analysis of Nonlinear Systems. In: Proceedings of ECC'97, Bruxelles, Belgium.

Slupphaug, Olav and Bjarne A. Foss (1998a). Bilinear Matrix Inequalities and Robust Stability of Nonlinear Multi-Model MPC. In: To appear in the Proceedings of the American Control Conference. Philadelphia, PA, USA.

Slupphaug, Olav and Bjarne A. Foss (1998b). Quadratic Stabilization of Discrete-Time Uncertain Nonlinear Multi-Model Systems using Piecewise Affine StateFeedback. Submitted to International Journal of Control. http://www.itk.ntnu.no/ansatte/Foss_Bjarne/publikasjoner/conference.html.

Tuan, H. D., S. Hosoe and H. Tuy (1997). D.C. Optimization Approach to Robust Control: Feasibility Problems. Technical Report 9601. Nagoya University, Japan. To be submitted for journal publication. 